\title{
Fomentar la argumentación en clases de ciencias a través de una controversia sociocientífica en futuros docentes
}

\author{
Natalia Jiménez-Tenorio, Juan José Vicente-Martorell, Lourdes Aragón \\ y José Ma Oliva-Martínez
}

Facultad de Ciencias de la Educación. Departamento de Didáctica, Área de Didáctica de las Ciencias Experimentales. Universidad de Cádiz. Puerto Real. España.

[Recibido el 31 de octubre de 2018, aceptado el 24 de octubre de 2019]

\begin{abstract}
La argumentación es una competencia que se debería trabajar de manera explícita y con profundidad en los procesos de enseñanza-aprendizaje de las ciencias. El propósito de este trabajo es evaluar la calidad de los argumentos que presentan futuros maestros de primaria en una actividad de una propuesta didáctica de corte argumentativa. La actividad fue un debate y la controversia sociocientífica trabajada "¿son las telefonías móviles perjudiciales para la salud?". La información recogida procedió de la argumentación individual. Éstas se analizaron utilizando el método de cluster, basado en el modelo de análisis s de argumentación de Toulmin. Los resultados muestran que la mayoría del alumnado se encuentra en niveles medios de argumentación. Un documento técnico sobre el pensamiento crítico y un blog fueron las fuentes más útiles para formar la opinión del alumnado. Consideramos la necesidad de seguir avanzando desde el ámbito universitario el desarrollo de propuestas en esta dirección.
\end{abstract}

Palabras clave: Argumentación; estrategia didáctica; modelo de Toulmin; campos electromagnéticos; formación inicial de profesorado.

\section{Encouraging argumentative discourse in science classes for future teachers by introducing controversial socio scientific topics}

To be able to expose an argument is a task that should be deeply covered during the teachinglearning process. The purpose of this paper is to evaluate the quality of the arguments presented by future primary school teachers in a socio-scientific debate activity. The proposed activity object of the experimentation was based on the question: 'are the mobile telephone antennas tower a risk for your health?'. The information was collected through individual reports that were analyzed using a cluster method applying the Toulmin's argument pattern. The results show us a level medium most of the students. A technical paper on critical thinking and a blog were the most useful sources for forming student opinion. We consider the necessity to continue reinforcing the development of experiences in the direction of the proposed activity in graduate programs.

Keywords: Argumentation; didactic strategy; Toulmin's argument pattern; electromagnetic fields; teacher initial training.

Para citar el artículo. Jiménez-Tenorio, N., Vicente-Martorell, J.J, Aragón, L. y Oliva-Martínez, J.M. (2020). Fomentar la argumentación en clase de ciencias a través de una controversia sociocientífica en futuros docentes. Ápice. Revista de Educación Científica, 4(1), 79-86. DOI: https://doi. org/10.17979/arec.2020.4.1.4639

Contacto.natalia.jimenez@uca.es, juanjose.vicente@uca.es, lourdes.aragon@uca.es, josemaria. oliva@uca.es 


\section{Planteamiento del problema}

El tema de la telefonía móvil y la instalación de antenas para obtener mayor cobertura y servicio a la ciudadanía suponen una oportunidad para estimular a los estudiantes y mejorar la toma de decisiones sobre problemas sociocientíficos, tema en auge en la enseñanza de las ciencias (Cebrián-Robles y Rodríguez-Mora, 2018). Sin embargo, en nuestras clases hemos observado grandes dificultades para expresar una opinión oral y/o escrita, y más cuando se trata de cuestiones científicas. El alumnado, además, manifiesta un escaso contacto con esta disciplina, tanto a nivel académico como en su vida cotidiana. En este sentido, experiencias como la que se muestra en este trabajo puede suponer una oportunidad para acercar este tipo de cuestiones a sus vidas y promover una adecuada alfabetización científica a través del desarrollo del pensamiento crítico sobre asuntos científico-tecnológicos y sobre este problema en particular.

\section{Marco teórico}

El lenguaje forma parte de nuestra vida y es fundamental para la comprensión, aprendizaje y adquisición de nuevos contenidos. Posee un papel primordial en los procesos de enseñanza-aprendizaje y en la construcción social del conocimiento. Asimismo, en la ciencia la comunicación y el lenguaje juegan también un importante papel en la creación de conocimiento científico, y desde la didáctica de las ciencias se reconoce la importancia de las prácticas discursivas para su aprendizaje. Por ello, la argumentación es considerada uno de los principales pilares en el aprendizaje de las ciencias (Erduran y JiménezAleixandre, 2008) en correspondencia con las prácticas científicas de indagación y modelización (Jiménez-Aleixandre, 2012).

Las cuestiones sociocientíficas son problemas reales, cercanos al ciudadano, abiertos, complejos y potencialmente controvertidos, que pueden ser analizadas bajo multitud de perspectivas ya que surgen de la interrelación entre ciencia, tecnología y sociedad (Jiménez-Aleixandre, 2010). Por tanto, la argumentación sobre ellas conlleva una visión multidisciplinar ya que se debe considerar una amplia variedad de dimensiones, informaciones y puntos de vista.

La utilización de las controversias y de la argumentación en el aula de ciencia promueve el desarrollo de la competencia científica ya que desarrolla la utilización de pruebas científicas para elaborar, comunicar conclusiones y para identificar los supuestos, pruebas y razonamientos que las sustentan (OCDE, 2006). Por otra parte, los debates con implicaciones en diversos aspectos de la vida de las personas (ético, medioambiental o de salud) favorecen el intercambio de opiniones, la generación de comentarios adecuados o el enriquecimiento del diálogo (Campaner y De Longhi, 2007). Sin embargo, se han registrado problemas en la capacidad de argumentación del alumnado sobre cuestiones sociocientíficas (Kolstø, 2006), por lo que Brocos, Jiménez y Rodríguez (2018) sugieren dar al alumnado un marco de toma de decisiones que facilite la valoración de diversas alternativas y mejore la calidad de sus argumentaciones sobre el problema en cuestión. Asimismo, algunas investigaciones apuntan que, en general, los profesores poseen dificultades para provocar escenarios argumentativos en sus clases de ciencias predominando otra serie de actividades (Archila, Luna-Calderón y Mesa-Piñeros, 2017).

Existen numerosas investigaciones sobre la argumentación desde el campo de la lingüística en general (Camps y Dolz, 1995) y de la ciencia en particular (Jiménez-Aleixandre y Díaz, 2003), que evalúan desde diferentes miradas la calidad de la argumentación, dependiendo del contexto en el que se produce (Maguregi, Uskola y Burgoa, 2017). Sin embargo, la mayoría de los autores para el análisis estructural de los textos argumentativos de ciencias se basan en el modelo de Toulmin (1958). Éste construye un modelo atendiendo a los 
diferentes elementos y las relaciones que estos pueden contener en una argumentación. Es por ello que se convierte en una herramienta útil para analizar la escritura de cualquier texto. De hecho, Rodríguez (2004) opina que se trata de un modelo aplicable incluso a argumentos de tipo social.

En este contexto, entendemos que debemos enseñar la argumentación a través de la práctica. Opinión compartida por Archila et al. (2017) que afirman que fomentar la argumentación debe ser una meta de la educación en ciencias. Por ello, es importante plantear situaciones de aprendizaje en las que los estudiantes discutan, y tareas que requieran un papel activo de los mismos elaborando textos y discusiones argumentativas científicas, es decir, fomentar "hablar ciencia" y "escribir ciencia" en el aula (Sanmartí, 2007). En esta línea, se pretende formar a nuestros estudiantes, futuros maestros de ciencias, en argumentación.

\section{Escenario formativo}

Este estudio se enmarca en un proyecto de innovación y mejora docente desarrollado durante el curso 2017-2018 en la asignatura de carácter obligatoria de Didáctica de las Ciencias de la Naturaleza I ( 6 créditos) de 3 o del Grado en Educación Primaria (GEP) de la Universidad de Cádiz (UCA, 2013).

El objetivo principal del proyecto fue desarrollar la argumentación de los estudiantes en contextos sociocientíficos a partir de actividades que implicasen evaluar enunciados, reflexionar, comunicar y relacionar datos y conclusiones. Para promover en los estudiantes la argumentación de forma sistémica y con un enfoque más estructurado, se diseñaron una serie actividades distribuidas en cada uno de los bloques que forman la asignatura y en las que, a través de ellas, los alumnos hacen y aprenden ciencia, a la vez que hablan y escriben de ciencia. La experiencia presentada en este trabajo se encuadra en el primer bloque de la asignatura donde se abordan las finalidades y problemáticas de la educación científica en la Educación Primaria.

\section{Diseño de la investigación}

El objetivo de este trabajo es analizar el nivel de complejidad de la argumentación del alumnado al inicio de la asignatura y antes de realizar alguna experiencia formativa para trabajar dicho aspecto. Asimismo, se pretende cuantificar el orden de prioridad en función de la utilidad que el alumnado da a los documentos de trabajo para conformar su opinión sobre el tema.

La investigación se enmarca en la fase de diagnósticos de la propuesta didáctica, que permite contextualizar y detectar cómo argumentan nuestros alumnos. El estudio se ha llevado a cabo en uno de los tres grupos-clases del 3er curso del GEP, integrado por 41 alumnos que se organizaron en 12 equipos de trabajo constituidos por 3 o 4 alumnos cada uno.

La actividad objeto de análisis se desarrolló en dos sesiones de trabajo (3h). La noticia aparecida en prensa "Los Villares dice 'no' a las antenas para la telefonía móvil (El país, 23 de agosto 2007 “https://elpais.com/tecnologia/2007/08/23/actualidad/1187857685_850215. html") sirvió como punto de partida para plantear la siguiente controversia sociocientífica: “¿son las telefonías móviles perjudiciales para la salud?”. Los equipos de trabajo, tras consultar varios documentos facilitados por el docente con distintos puntos de vista sobre el dilema (tabla 1), llegaron a un consenso sobre la peligrosidad de los teléfonos móviles y sus antenas para la salud. Posteriormente, se realizó un debate en gran grupo, enfrentándose ambas posiciones, finalizando con la votación de cada grupo para tomar la decisión de instalar o no la antena. 
Tabla 1. Documentos utilizados para el desarrollo de la actividad

\begin{tabular}{|c|c|}
\hline $\begin{array}{l}\text { Tipo de documento } \\
\text { Documento alojado en la web www.escepticos.es } \\
\text { escrito por el Dr. John Moulder en } 1995 \text { bajo } \\
\text { contrato con la ciudad de Brookfield, Wisconsin } \\
\text { (Estados Unidos), mantenido y expandido desde } \\
1995 \text { como apoyo para la enseñanza en el Medical } \\
\text { College of Wisconsin }\end{array}$ & $\begin{array}{l}\text { Título } \\
\text { "Preguntas y respuestas sobre antenas de } \\
\text { telefonía móvil" }\end{array}$ \\
\hline Noticia aparecida en la Voz de Galicia & $\begin{array}{l}\text { "El cargador del móvil provoca un campo } \\
\text { electromagnético que afecta a la cabeza" }\end{array}$ \\
\hline Noticia aparecida en El Mundo & $\begin{array}{l}\text { "La OMS reconoce una posible relación entre } \\
\text { los móviles y algunos tipos de cáncer" }\end{array}$ \\
\hline Hoja descriptiva de la OMS no 296 & "Campos electromagnéticos y salud pública" \\
\hline $\begin{array}{l}\text { Entrada aparecida en el blog de ciencia del } \\
\text { periódico Libertad Digital }\end{array}$ & "Móviles, cáncer y la OMS (para variar)" \\
\hline $\begin{array}{l}\text { Información publicada en la web del Instituto } \\
\text { Nacional del Cáncer ( } \mathrm{NCl} \text { ) de los EE. UU }\end{array}$ & "Teléfonos celulares y el riesgo de cáncer" \\
\hline Documento de la AA.VV. Bajo Albayzín (Granada) & "Antenas de telefonia" \\
\hline
\end{tabular}

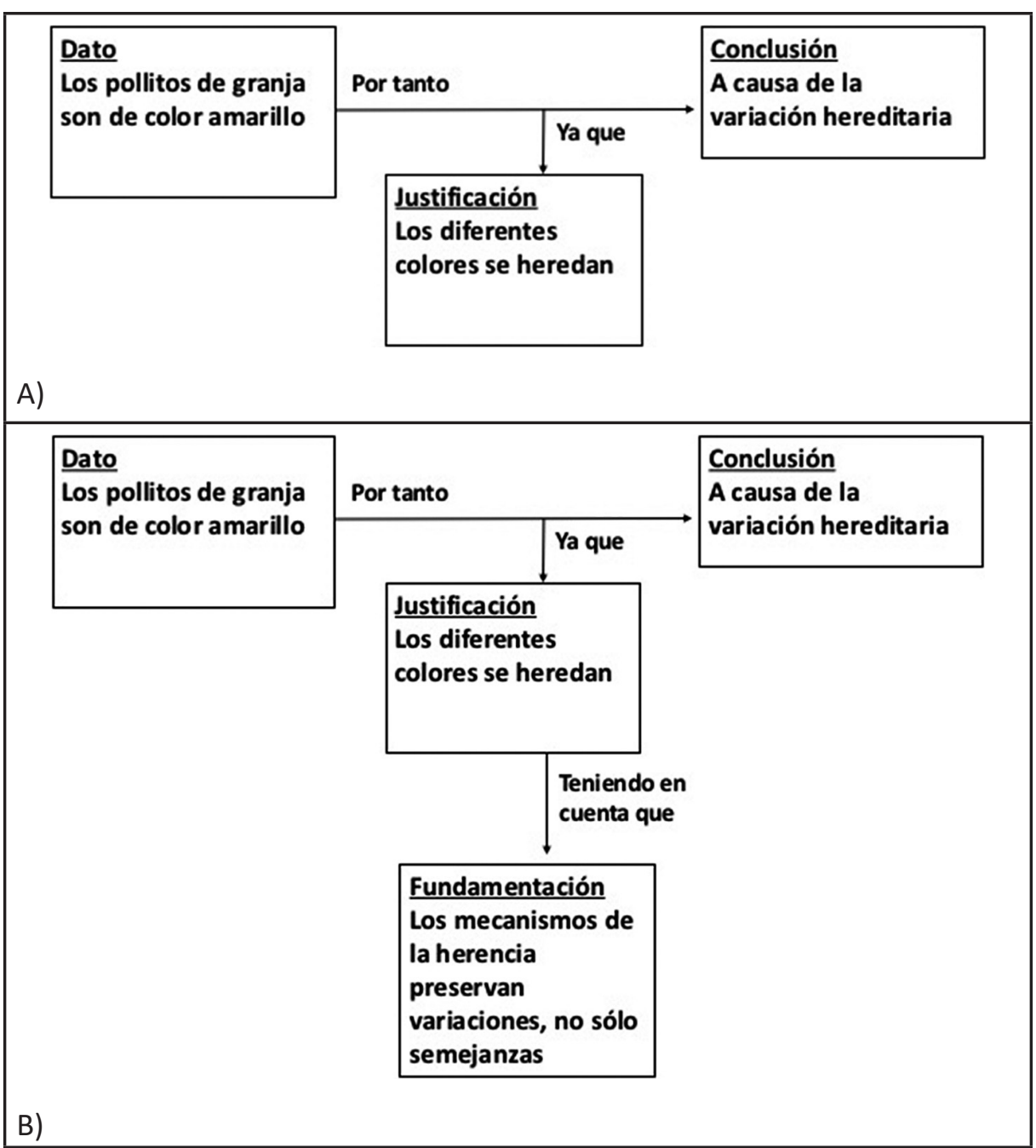

Figura 1. Ejemplos de niveles de argumentación según el método clúster: A) de orden III (DJC), y B) de orden IV (DJFC) (Fuente: Jiménez Aleixandre, 2010) 
Las producciones de la actividad consistieron en un informe que contiene: la argumentación del voto y el orden de importancia dado a los documentos consultados por cada alumno, el voto consensuado y argumentado del grupo, y un audio grabado durante todo el proceso de discusión y toma de decisión del grupo.

Para medir la complejidad de la argumentación inicial del alumnado, se analizaron las explicaciones de los votos individuales de los 41 alumnos. Para ello, se llevó a cabo un análisis cuantitativo utilizando el método cluster (Erduran, Simon y Osborne, 2004). Éste consiste en un análisis del discurso identificando distintos elementos que se encuentran conectados a través de relaciones lógicas concretas, para posteriormente, clasificar la argumentación en base a la diversidad de elementos utilizados.

Este método, basado en el modelo de Toulmin, identifica los siguientes elementos en una argumentación: datos (D), justificación (J), razones o argumentos $(R)$, fundamentación $(F)$, refutación u objeciones (Ref), validez (V) y conclusiones (C). La estructura mínima es aquella que está formada por los tres elementos básicos "DJC", siendo ésta considerada como una argumentación de nivel III (ejemplo en la figura 1). A partir de aquí, en función del número de elementos presentes, subirá de nivel de complejidad de la argumentación, siendo la estructura más compleja aquella que incluya los siete elementos (nivel VII: DJRFRefVC).

El nivel de argumentación de los estudiantes fue analizado de manera conjunta por dos de los autores del trabajo, que consensuaron posiciones y decisiones. Cuando no existía acuerdo entraba en juego el tercer autor. Por otra parte, se cuantificó el orden de las fuentes consultadas para conocer la utilidad otorgada por el alumnado a estas para construir sus argumentos y tomar su decisión.

\section{Resultados y discusión}

Los resultados obtenidos del análisis de las argumentaciones elaboradoras por los alumnos en la actividad planteada se muestran en la figura 2. En ella se observan los distintos niveles de argumentación presentados por los estudiantes, en base al número de elementos que componen sus argumentos. Se observa que los mayores porcentajes de alumnos se encuentran en los niveles IV y V de argumentación con un $31,7 \%$ y un $36,6 \%$, respectivamente. El 15,2\% del alumnado presenta un nivel de argumentación de orden III, siendo menos frecuente encontrar argumentos de orden VI y VII.

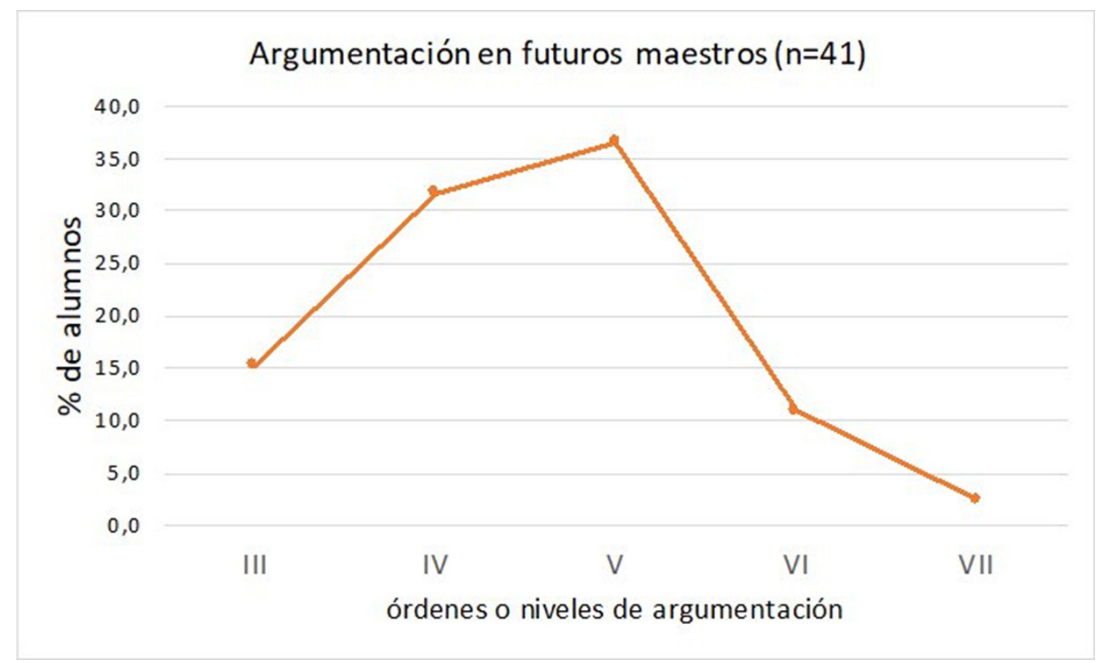

Figura 2. Resultados obtenidos del nivel de complejidad en las argumentaciones estimadas mediante el método cluster (Fuente: elaboración propia) 
Por otro lado, con respecto a los niveles de argumentación más frecuentes encontrados en el alumnado se encuentran distintas combinaciones. De las 15 argumentaciones cuantificadas de orden $\mathrm{V}$, los cinco elementos se combinan de varias maneras posibles, aunque predominan algunas con respecto a otras. Así, 5 de ellas contienen los elementos "DJFRefC" (figura 3A) y otras 5 presentan la combinación "DJFVC" (figura 3B).

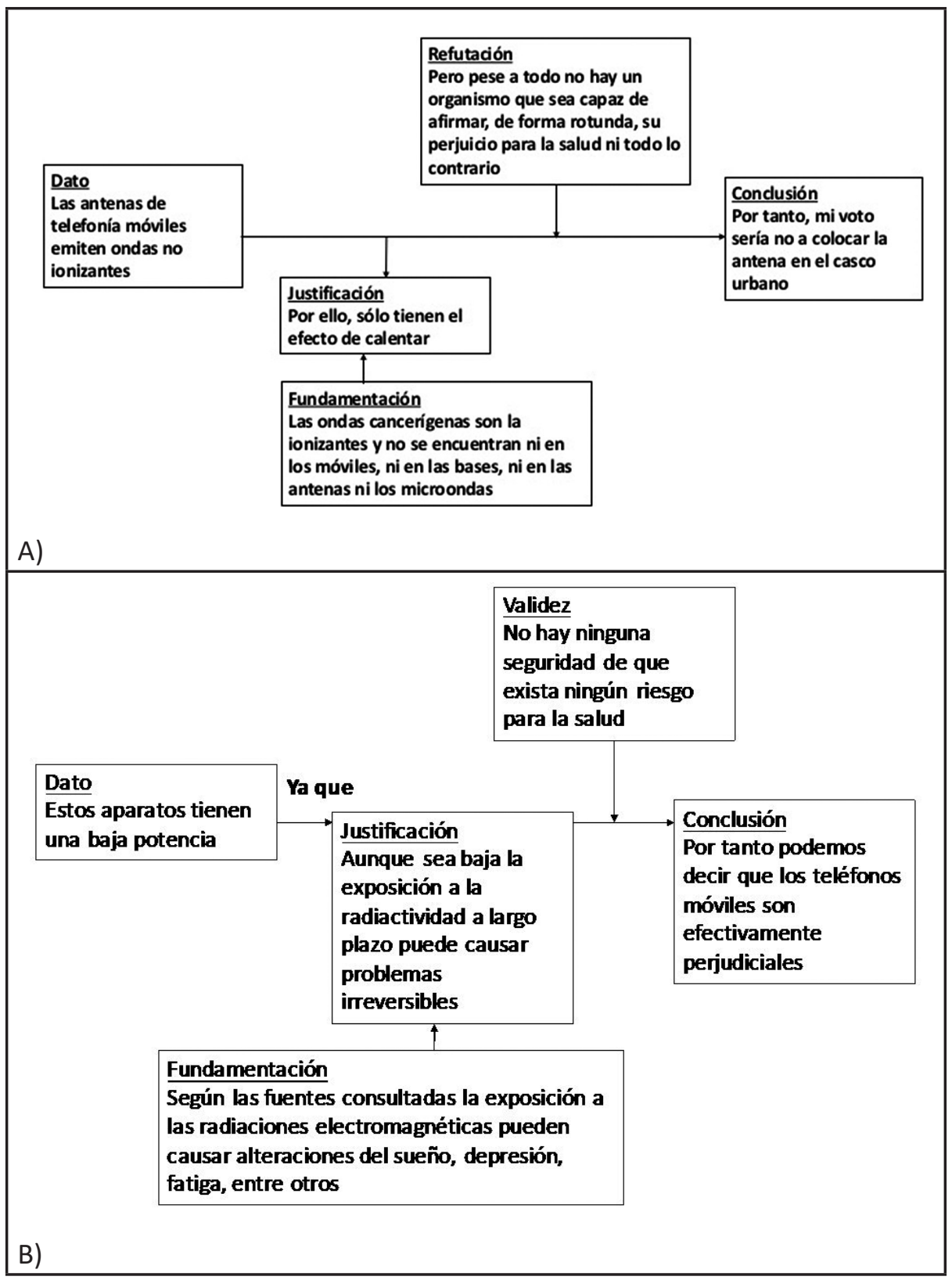

Figura 3. Ejemplo de argumento expuesto por los estudiantes de orden $V$ en la combinación: A) DJFRefC, y B) DJFVC

En relación al orden de prioridad de las fuentes consultadas por los estudiantes, se observa que el primer puesto lo ocupa un documento técnico incluido en la página web escépticos.es. En segundo lugar, los alumnos emplean una entrada en el blog de ciencia del periódico Libertad Digital. Ambas fuentes incluyen información relativa a que las investigaciones realizadas hasta el momento no han conseguido demostrar que exista relación entre la telefonía móvil y riesgos para la salud como el cáncer. 
Los siguientes documentos en orden de importancia han sido la hoja informativa de la OMS en la que se decide incluir los campos de frecuencia electromagnética, como los que desprenden los móviles, en la categoría de compuestos posiblemente carcinógenos para los humanos, y la noticia aparecida en prensa que informa de esta decisión. Es destacable que la hoja informativa publicada en la web del Instituto Nacional del Cáncer ( $\mathrm{NCl}$ ) de los EE.UU aparece en el quinto lugar de esta lista. El último lugar lo ocupa el documento que recoge la oposición de un barrio de Granada a la instalación de telefonía. Estos resultados hacen pensar que los alumnos dan una mayor credibilidad y valor a aquellas fuentes que refuerzan sus ideas preconcebidas sobre el tema, dando prioridad a aquellas que transmiten el mensaje de una manera más directa y clara.

\section{Conclusiones}

Consideramos que este tipo de actividades planteadas en la formación inicial del profesorado potencia el pensamiento crítico, pero sobre todo, acercar al alumnado a estrategias de aula que conlleve utilizar el conocimiento científico para tomar una decisión cercana a su vida diaria. Además, se encuentran en consonancia con la visión actual de la enseñanza de las ciencias, como es formar a ciudadanos críticos y reflexivos ante la información que reciban, y competentes para ofrecer opiniones argumentadas a la hora de tomar decisiones en su vida diaria y esperemos tengan repercusión, en sus futuras acciones como docentes. Sin embargo, somos conscientes de la necesidad de seguir indagando en secuencias didácticas donde la argumentación esté más presente y plantear, desde la asignatura del GEP, estrategias que promuevan aún más la argumentación. En esta línea, algunos estudios muestran que los estudiantes valoran muy positivamente los debates sociocientíficos llegando incluso a conseguir un cambio de actitud en ellos, mejorando su rendimiento académico (Ruiz, Solbes y Furió, 2003).

\section{Agradecimientos}

Financiado por: FEDER/Ministerio de Ciencia, Innovación y Universidades-Agencia Estatal de Investigación/ Proyecto EDU2017-82518-P.

\section{Referencias bibliográficas}

Archila, P.A., Luna-Calderón, P. y Mesa-Piñeros, M. (2017). El empleo espontáneo de conectores y vocabulario relacionado con las ciencias: Implicaciones en la argumentación escrita. Revista Eureka sobre Enseñanza y Divulgación de las Ciencias, 14(1), 3-23. Recuperado de: http://hdl.handle.net/10498/18843

Brocos, P., Jiménez, M.P. y Rodríguez, R. (2018). Diseño e implementación de un proyecto multidisciplinar sobre argumentación y alimentación en Bachillerato. En C. Martínez Losada, S. García Barros (Eds.), 28 Encuentros de Didáctica de las Ciencias Experimentales (pp. 115-119). A Coruña: Universidade da Coruña y APICE. DOI: https://doi.org/10.17979/spudc.9788497496896

Campaner, G. y De Longhi, A.L. (2007). La argumentación en Educación Ambiental. Una estrategia didáctica para la escuela media. Revista Electrónica de Enseñanza de las Ciencias, 6(2), 442-456. Recuperado de: http://reec.educacioneditora.net/

Camps, A. y Dotz, J. (1995). Introducción: enseñar a argumentar, un desafío para la escuela actual. Comunicación, Lenguaje y Educación, 25, 5-8.

Cebrián-Robles, D. y Rodríguez-Mora, F. (2018). Diseño de actividades de argumentación científica sobre el consumo de agua embotellada. Propuesta de evaluación mediante 
rúbricas. En C. Martínez Losada, S. García Barros (Eds.), 28 Encuentros de Didáctica de las Ciencias Experimentales (pp. 637-642). A Coruña: Universidade da Coruña y APICE. DOI: https://doi.org/10.17979/spudc.9788497496896

Erduran, S. y Jiménez-Aleixandre, M.P. (2008). Argumentation in science education. Berlin: Springer.

Erduran, S., Simon, S. y Osborne, J. (2004). TAPping into Argumentation: Developments in the Application of Toulmin's Argument Pattern for Studying Science Discourse. Science Education, 88, 915-933. Recuperado de: http://discovery.ucl.ac.uk/id/ eprint/10000654

Kolst $\varnothing$, S. D. (2006). Patterns in students' argumentation confronted with a risk-focused socioscientific issue. International Journal of Science Education, 28(14), 1689-1716. DOI: https://doi.org/10.1080/09500690600560878

Jiménez-Aleixandre, M. P. (2010). 10 Ideas Clave. Competencias en argumentación y uso de pruebas. Barcelona: Graó.

Jiménez-Aleixandre, M.P. (2012). Las prácticas científicas en la investigación y en el aula de ciencias. Conferencia plenaria. En J.M. Domínguez (Ed.), XXV Encuentros de Didáctica de las Ciencias Experimentales (pp. 9-14). Santiago de Compostela: Universidade de Santiago de Compostela y APICE.

Jiménez-Aleixandre, M.P. y Díaz, J. (2003). Discurso de aula y argumentación en la clase de ciencias: cuestiones teóricas y metodológicas. Enseñanza de las ciencias, 21(3), 359-370. Recuperado de: https://core.ac.uk/download/pdf/38990750.pdf

Maguregi, G., Uskola, A. y Burgoa, B. (2017). Modelización, argumentación y transferencia de conocimiento sobre el sistema inmunológico a partir de una controversia sobre vacunación en futuros docentes. Enseñanza de las Ciencias, 35(2), 29-50. DOI: http://dx.doi.org/10.5565/rev/ensciencias.2237

OCDE (2006). PISA 2006. Marco de la evaluación: Conocimientos y habilidades en Ciencias, Matemáticas y lectura. Madrid: Santillana Educación S.L.

Rodríguez, L.I. (2004). El modelo argumentativo de Toulmin en la escritura de artículos de investigación educativa. Revista Digital Universitaria, 5(1), 2-18. Recuperado de: http://www.revista.unam.mx/vol.5/num1/art2/art2.htm

Ruiz, J.J., Solbes, J., y Furió, C. (2010). Los debates sociocientíficos: un recurso para potenciar la competencia argumentativa en las clases de física y química. Enseñanza de las ciencias, Núm. Extra, 3126-3131. Recuperado de: https://www.raco.cat/index.php/ Ensenanza/article/view/308283.

Sanmartí, N. (2007). Hablar, leer y escribir para aprender ciencia. En P. Fernández (Coord.), La competencia en comunicación lingüística en las áreas del currículo (pp. 103-128). Madrid: MEC, Colección Aulas de Verano.

Toulmin, S. (1958). The use of argument. Cambridge: University Press.

Universidad de Cádiz (UCA). (2013) Memoria del Título del Grado en Educación Primaria. Recuperado de: http://www.uca.es/recursosgen/doc/Centros/ciencias_educacion/ grado_primaria/368495804_722014142951.pdf 